\title{
High-repetition-rate femtosecond- laser micromachining of low-loss optical-lattice-like waveguides in lithium niobate
}

Teerawat Piromjitpong, Mykhaylo Dubov, Sonia Boscolo

Teerawat Piromjitpong, Mykhaylo Dubov, Sonia Boscolo, "High-repetitionrate femtosecond-laser micromachining of low-loss optical-lattice-like waveguides in lithium niobate," Proc. SPIE 10684, Nonlinear Optics and its Applications 2018, 106840D (14 May 2018); doi: 10.1117/12.2306643

SPIE. Event: SPIE Photonics Europe, 2018, Strasbourg, France 


\title{
High-repetition-rate femtosecond-laser micromachining of low-loss optical-lattice-like waveguides in lithium niobate
}

\author{
Teerawat Piromjitpong ${ }^{*}$, Mykhaylo Dubov ${ }^{\mathrm{b}}$, and Sonia Boscolo ${ }^{\mathrm{a}}$ \\ ${ }^{a}$ Aston Institute of Photonic Technologies, School of Engineering and Applied Science, Aston \\ University, Birmingham B4 7ET, UK; ' $O$ Optoscribe Ltd., Livingston EH54 7EJ, UK
}

\begin{abstract}
A series of waveguides were inscribed in lithium niobate by tightly focused femtosecond-laser pulses of 11-MHz repetition rate and 790-nm wavelength. To establish the inscription conditions for optimal low-loss waveguides, within each sample we varied laser pulse energy, speed and direction of translation stage movement, and focus depth of the beam. We deployed two new approaches to enhance the inscription results: 1) increase of the pulse energy with increasing focus depth inside the material to compensate for the corresponding decrease of refractive-index modification, and 2) decrease of the laser energy for the modification tracks closer to the waveguide's core region to reduce scattering losses due to high-laser-energy driven non-uniformities. All waveguides had an optical-lattice-like hexagonal packing geometry with track-spacing of $9.9 \mu \mathrm{m}$ (optimized for effective suppression of high-order modes). Each structure comprised 84 single-scan Type-II-modification tracks, aligned with the crystalline X-axis of lithium niobate. After heat treatment at $350{ }^{\circ} \mathrm{C}$ for 3 hours, the lowest propagation loss of less than $(0.4 \pm 0.1) \mathrm{dB} / \mathrm{cm}$ and $(3.5 \pm 0.3) \mathrm{dB} / \mathrm{cm}$ for the ordinary and extraordinary light polarization states, respectively, were achieved at the 1550$\mathrm{nm}$ wavelength. These low-attenuation waveguides were obtained with the inscription energy varying between 50.6 $\mathrm{nJ}$ and $53.6 \mathrm{~nJ}$ and the translation speed of $10 \mathrm{~mm} / \mathrm{s}$. The corresponding refractive-index contrast of individual tracks was $(-1.55 \pm 0.04) \times 10^{-3}$. The waveguides also showed low attenuation in the visible and near-infrared portion of the spectrum $(532 \mathrm{~nm}$ to $1456 \mathrm{~nm}$ ). Our results offer promising means for the development of low-loss waveguides with preserved-nonlinearity and high thermal stability.
\end{abstract}

Keywords: femtosecond laser inscription, high-repetition-rate femtosecond laser, lithium niobate, optical-lattice-like waveguide, nonlinear waveguide

*piromjit@aston.ac.uk

\section{INTRODUCTION}

Lithium niobate (LN) offers incredible versatility as a substrate for integrated photonics (IP) owing to its wide range of transparency, high second-order nonlinear coefficient, and commercial availability ${ }^{1}$. Recently, a number of LN-based IP devices have been demonstrated, such as electro-optic modulators ${ }^{2,3}$ and various types of frequencymixing devices ${ }^{4,5}$, which show a potential for applications in high-speed optical communication interconnects, on-chip sensors, and quantum photonics.

Among the various micro-structure fabrication technique of photonic waveguides, such as proton exchange, Ti indiffusion, iron implantation and dry etching, the femtosecond (fs) laser micromachining method is considered to offer improved versatility in the choice of processed materials and greater flexibility in creating micro-structures in the three-dimensional space ${ }^{6,7}$. The technique relies on tightly focusing a fs-pulse-width light beam into a sample of a transparent dielectric so that to induce one or more material modifications (e.g., valence electron shift, chemical composition change, or amorphization) at the beam focal spot via multi-photon absorption and/or ionization processes $^{8}$.

In general, fs-laser written waveguides in $\mathrm{LN}$ can be categorized into three groups depending on the mechanism of optical guiding: positive-refractive-index(RI)-induced ${ }^{9-13}$, stress-induced ${ }^{14-18}$, and depressed-cladding waveguides ${ }^{14,19-24}$. The focus of this study is on the fabrication and optimization of waveguides of the third type, which present a number of advantages over the first two types, including higher thermal stability, output-profile controllability and low input-polarization dependence. Furthermore, our deployment of a high-repetition-rate (HRR) fs-laser enables relatively shorter processing times compared to its low-repetition-rate (LRR) counterpart. This is especially advantageous to the fabrication of complex structures and large-scale commercial production. We explored the effects of operational parameters such as the laser pulse energy, inscription translation speed and direction and

Nonlinear Optics and its Applications 2018, edited by Benjamin J. Eggleton, Neil G. R. Broderick, Anna C. Peacock Proc. of SPIE Vol. 10684, 106840D · (C) 2018 SPIE · CCC code: 0277-786X/18/\$18 · doi: 10.1117/12.2306643 
beam focus depth on the inscription process, and we also investigated the post-fabrication thermal annealing process. Two new approaches based on varying the inscribing pulse energy in accordance with the position of the tracks were implemented to enhance the inscription results, and the resulting waveguides were compared with a reference set of waveguides. To the best of our knowledge, this work is the first to demonstrate low loss waveguides for both the ordinary (TE) and extraordinary (TM) polarization states using HRR fs-laser micromachining with LN. The heat treatment also revealed an interesting feature where the RI change of the inscribed track was enhanced by the process and remained stable up to a temperature of $700{ }^{\circ} \mathrm{C}$.

\section{EXPERIMENTAL SETUP AND PROCEDURES}

The inscribing laser was a mirror-dispersion-controlled Ti:Sapphire oscillator system (Femtosource Scientific XL, Femtolasers) that delivered an output beam at $790-\mathrm{nm}$ central wavelength and 11-MHz repetition-rate. The pulse duration was adjusted to $66 \mathrm{fs}$, corresponding to a spectral width of approximately $25 \mathrm{~nm}$. The oscillator was pumped by a 532-nm solid-state diode-pump continuous-wave laser (Verdi V-10, Coherent), which provided 7.5-W optical power. For this pump power, the pulse energy was up to $91 \mathrm{~nJ}$. A motorized half-wave plate and a pair of Brewster angle polarizers were used to control the inscribing pulse energy, and a quarter-wave plate was used to convert the beam from linear to circular polarization before it was delivered to a focusing objective. As depicted in Figure 1a, a 100x microscope objective with a numerical aperture of 1.25 was used to focus the beam beneath the sample surface at a maximum depth of $0.4 \mathrm{~mm}$ (limited by the objective's working distance). The samples were polished $5 \%$ magnesium-doped z-cut LN wafers of 75-mm diameter and 1-mm thick. They were mounted on a two-dimensional micro-positioning air-bearing stage (Aerotech), which enabled sub-micrometer resolution translations along the horizontal axes at a maximum speed of $100 \mathrm{~mm} / \mathrm{s}$. The combination of horizontal sample translation with a synchronous movement of the objective along the vertical direction by a mechanical stage enabled the creation of three-dimensional waveguide structures. The immersion oil layer $(\mathrm{RI}=1.523)$ between the lenses and the sample was checked regularly during the experiment to prevent any formation of air-bubbles or dust that could affect the inscription results. A detailed description of our laser inscription system can be found in Dubov et al. ${ }^{25}$.

Figure $1 \mathrm{~b}$ shows an example of a waveguide cross-section, where the circle array represents the track positions programmed into the translational stage controller and the inset shows the actual track positions and shapes after fabrication. Each waveguide comprised 84 tracks precisely arranged in an optical-lattice-like packing geometry with four hexagonal layers. The tracks were damage tracks or of so-called 'Type-II' modification ${ }^{26}$, and were inscribed via a single scan along the LN crystalline $\mathrm{X}$-axis. After preliminary trials with different track spacing values, we found that a separation between $9 \mu \mathrm{m}$ and $12 \mu \mathrm{m}$ is optimal in that the tracks are neither so densely packed to induce structural defects, nor so loosely packed to produce multi-mode optical confinement. In this study, we used a horizontal spacing $\left(d_{1}\right)$ of $9.9 \mu \mathrm{m}$, which resulted in a vertical spacing $\left(d_{2}\right)$ equaling an integer multiple of the step size of the vertical translation stage attached to the focusing microscope objective.

We observed that focusing the beam at different depths (D) inside the sample induced tracks with significantly different cross-sectional dimensions (width and height) and RI change, thereby leading to a vertically un-balanced waveguide's cladding. In fact, both the track size and RI change tended to decrease when the tracks were inscribed

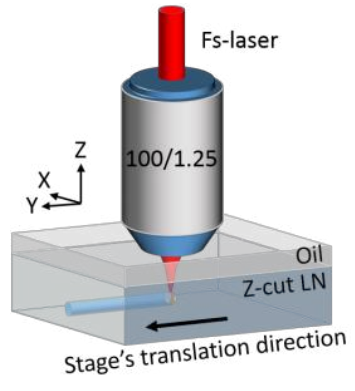

(a)

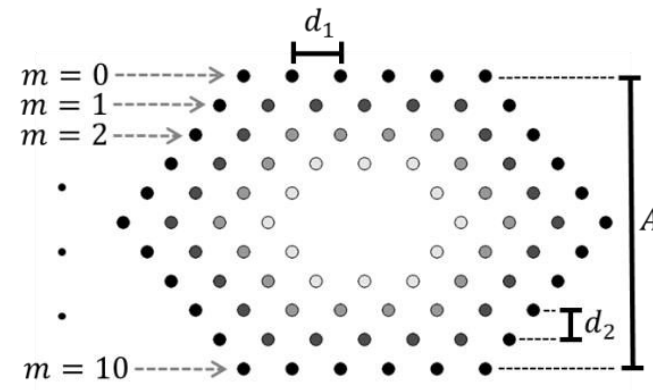

(b)

Figure 1. (a) Schematic of the experimental setup showing the alignment and relative movements of microscope objective and LN sample. (b) Cross-sectional configuration of a waveguide, where the circles represent the track positions written in the computer code, and the inset shows the actual track positions and shapes after inscription. 


\begin{tabular}{|c|c|c|c|c|c|c|c|c|c|c|c|c|c|c|c|c|}
\hline Group & & & & & & & & & & & & & $\begin{array}{l}\Delta E_{d} \\
(\mathrm{~nJ})\end{array}$ & $\begin{array}{l}\Delta E_{r} \\
(\mathrm{~nJ})\end{array}$ & $p$ & $\begin{array}{c}\mathrm{S} \\
(\mathrm{mm} / \mathrm{s})\end{array}$ \\
\hline \multirow{2}{*}{$\mathbf{A}$} & Name & - & A01 & A02 & A03 & A04 & A05 & A06 & A07 & A08 & A09 & A10 & \multirow{2}{*}{0.0} & \multirow{2}{*}{0.0} & \multirow{2}{*}{0.0} & \multirow{2}{*}{10} \\
\hline & $E_{0}(\mathrm{~nJ})$ & - & 39.1 & 40.9 & 42.7 & 45.5 & 48.2 & 50.9 & 53.6 & 56.4 & 59.1 & 61.8 & & & & \\
\hline \multirow{2}{*}{ B } & Name & B00 & B01 & B02 & B03 & B04 & B05 & B06 & B07 & B08 & B09 & B10 & \multirow{2}{*}{0.3} & \multirow{2}{*}{0.0} & \multirow{2}{*}{0.0} & \multirow{2}{*}{10} \\
\hline & $E_{0}(\mathrm{~nJ})$ & 37.3 & 39.1 & 40.9 & 42.7 & 45.5 & 48.2 & 50.9 & 53.6 & 56.4 & 59.1 & 61.8 & & & & \\
\hline \multirow{2}{*}{ BX } & Name & - & BX1 & BX2 & BX3 & BX4 & - & - & - & - & - & - & \multirow{2}{*}{0.3} & \multirow[t]{2}{*}{0.0} & \multirow[t]{2}{*}{0.0} & \multirow{2}{*}{7} \\
\hline & $E_{0}(\mathrm{~nJ})$ & - & 39.1 & 43.6 & 50.9 & 56.4 & - & - & - & - & - & - & & & & \\
\hline & Name & \multicolumn{11}{|c|}{$E_{0}(\mathrm{~nJ})$} & \multirow{2}{*}{\multicolumn{2}{|c|}{13.7}} & \multirow[b]{2}{*}{2.2} & \multirow{2}{*}{10} \\
\hline \multirow{3}{*}{ C } & C01 & \multirow{2}{*}{\multicolumn{11}{|c|}{$\begin{array}{l}40.9 \\
42.7\end{array}$}} & & & & \\
\hline & $\mathrm{CO2}$ & & & & & & & & & & & & 0.3 & 8.2 & 1.0 & 10 \\
\hline & $\mathrm{C03}$ & \multicolumn{11}{|c|}{40.9} & 0.3 & 13.7 & 1.0 & 10 \\
\hline
\end{tabular}

Table 1. Inscription parameters for different groups of fabricated waveguides.

deeper. To compensate for these depth-dependent effects, we suggest here a simple but effective approach, consisting in gradually increasing the pulse energy for increasing inscription depth. In this work, a fixed energy increase rate $\left(\Delta E_{d}\right)$ of approximately $0.3 \mathrm{~nJ}$ (corresponding to $3-\mathrm{mW}$ optical power) per distance between two consecutive track rows was chosen after investigating some try-out fabrications. While the induced RI change of a track increases linearly with the inscribing pulse energy ${ }^{25}$, which is crucial for obtaining strongly confining depressed-cladding waveguides, increased pulse energy is liable to induce structural defects and micro-crack generations along the track. These high-laser-energy driven non-uniformities would result in a reduction of the waveguide performance due to effects such as scattering, absorption, and so on. To avoid this pitfall, we suggest the application of a radial variation of the inscribing pulse energy such that less energy is set aside for the tracks closer to the waveguide's core. Hence if we denote by $\Delta E_{r}$ the radial energy increase step, the pulse energy $\left(E_{m, n}\right)$ allocated to the machining of each track can be described by the following equation:

$$
E_{m, n}=E_{0}+m \Delta E_{d}+\left(\frac{n}{3}\right)^{p}\left(\Delta E_{r}\right)
$$

Where $m$ and $n$ are the row and layer orders, respectively (see Figure 1b), $p$ is a power factor, and $E_{0}$ is an initial energy corresponding to the uniform case. The values of the energy variation parameters and translation speed (S) used to fabricate our waveguides are listed in Table 1, where we have classified the waveguides according to their inscription conditions.

After the waveguide fabrication, the LN wafers were diced to a width of $2.35 \mathrm{~cm}$ (the length of the waveguides) and side-polished to the optical grade by a Logitech polishing machine. An optical microscope (Zeiss Axioscope-2 MOT) was used to photograph the waveguides and individual tracks in the differential interference contrast (DIC) and bright field modes. A series of track's top-view images recorded at slightly different focus distances were used to calculate the cumulative phase profile by commercial software from IATIA using the quantitative phase microscopy (QPM) method. A two-dimensional profile of the RI change of individual tracks (with respect to the non-irradiated areas) was then reconstructed. From the RI change profile we retrieved the differences between the negative peak located closely to the irradiated area center and the adjacent positive humps at a number of different positions along each track, and we calculated the average of these RI differences, which hereafter we will refer to as the 'RI contrast' of a track.

The light sources for waveguide characterization included three fiber-coupled laser diodes from QPhotonics (QFBDLD-1064: $\lambda_{0}=1064 \mathrm{~nm}$, QFBDLD-1450: $\lambda_{0}=1456 \mathrm{~nm}$ and QDFBLD-1550: $\lambda_{0}=1547 \mathrm{~nm}$ ), an ILX Lightwave laser diode (MPS-8033: $\lambda_{0}=1310 \mathrm{~nm}$ ) and a Fianium SC450-4 supercontinuum source which operated with three bandpass filters from Semrock $\left(\lambda_{0}=531 \mathrm{~nm} \Delta \lambda \approx 25 \mathrm{~nm}, \lambda_{0}=700 \mathrm{~nm} \Delta \lambda \approx 20 \mathrm{~nm}\right.$, and $\left.\lambda_{0}=840 \mathrm{~nm} \Delta \lambda \approx 22 \mathrm{~nm}\right)$. As

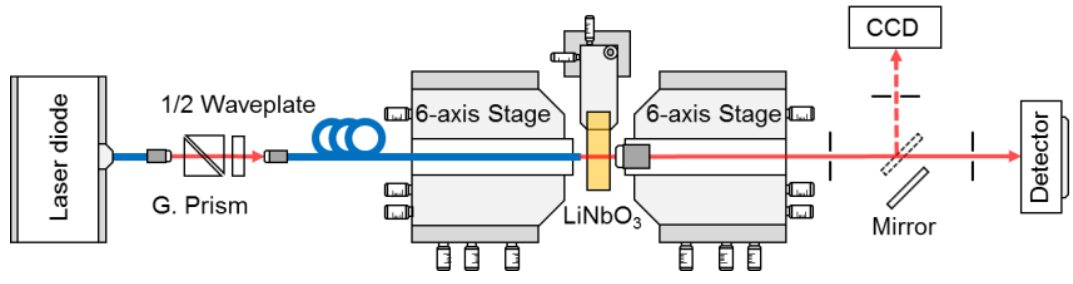

Figure 2. Schematic of the waveguide characterization setup. 
illustrated in Figure 2, a prism polarizer and a half-wave plate were used to adjust the polarization of the light from the source to either the TE mode (parallel to the LN's crystalline X-axis) or the TM mode (parallel to the LN's Z-axis) before coupling the light to a single-mode pigtail fiber. The beam was then butt-coupled into the waveguide being investigated by means of a 6-axis manually controlled stage, and the optical output from the waveguide was measured by a Germanium photodetector (Newport 818 -IR). The propagation loss $(\alpha)$ was estimated by subtracting the double Fresnel loss and the loss due to mode mismatch from the insertion loss. The mode-mismatch loss was evaluated by the overlap integral of the measured electric fields of the waveguide and the coupling single-mode fiber (mode field diameter $(\mathrm{MFD}) \approx 10.4 \mu \mathrm{m}$ at $1550 \mathrm{~nm}$ ). Subsequently, a sliding mirror was used to switch the beam path into an InGaAs infrared camera (Goodrich SUI SU320MS-1.7RT) to capture the near-field mode intensity distribution of the waveguide output.

A series of heat treatments of the waveguides were performed at the four temperatures of $250{ }^{\circ} \mathrm{C}, 350{ }^{\circ} \mathrm{C}, 500$ ${ }^{\circ} \mathrm{C}$ and $700{ }^{\circ} \mathrm{C}$ in an incremental order by using an open-air furnace. The annealing duration time was set to 3 hours, excluding heating-up and cooling-down periods, for each temperature. The heating-up and the cooling-down rates were approximately $1 \mathrm{~K} / \mathrm{s}$ and $3 \mathrm{~K} / \mathrm{s}$, respectively. The optical characteristics of the waveguides and the properties of individual tracks were thoroughly tested after each thermal annealing stage.

We also performed computer simulations of the waveguide structures by using the COMSOL commercial software based on the finite-element method (details can be found in Karakuzu et al. ${ }^{27}$ ). The packing geometry of the simulated waveguides was built so that to resemble the structure of the fabricated waveguides. The values of the RI contrast of the tracks and the waveguide cross-sectional sizes measured from the inscription parameter tests were inputted into the software to simulate the guided fundamental mode and calculate the MFD. To account for the overall effects of the stress field induced by individual tracks, we included in the model a hexagonally-shaped area with a slightly higher RI than the bulk material as a background layer tightly covering both the waveguide's core and cladding regions. The RI of this background layer was adjusted relative to the negative RI contrast assigned to the tracks.

\section{RESULTS AND DISCUSSION}

\subsection{Effects of Operational Parameters on Fs-Laser Modifications}

Like all our waveguides, the tracks were written by moving the sample relative to the pulse beam in the direction parallel to the $\mathrm{X}$-axis of $\mathrm{LN}$ crystal (either toward the positive $+\mathrm{X}$ or negative $-\mathrm{X}$ sign), where the beam itself propagated in the direction of the negative Z-axis. Figure 3a plots the RI contrast measured at 530-nm wavelength,

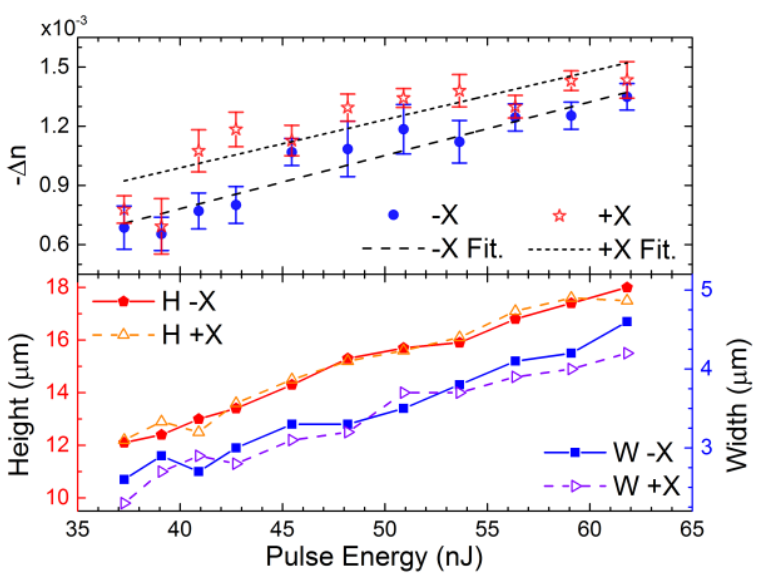

(a)

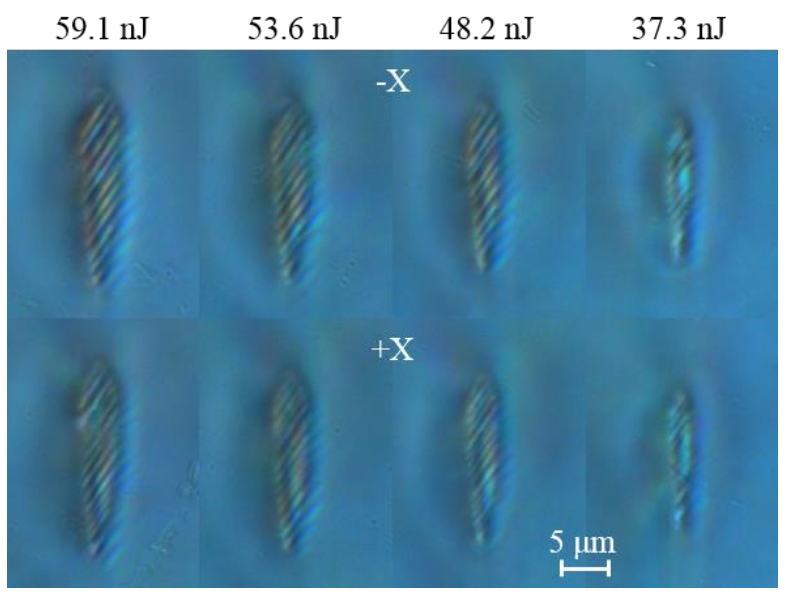

(b)

Figure 3. a) RI contrast measured at 530-nm wavelength (top), and the cross-sectional height and width (bottom) of the tracks as a function of inscribed pulse energy, where $\mathrm{S}=10 \mathrm{~mm} / \mathrm{s}$ and $\mathrm{D}=86 \mu \mathrm{m}$. The error bars represent the variation (standard deviation) in the RI contrast collected from ten different track positions. b) DIC images of some chosen tracks from the left plots. Top- and bottom-row images are the tracks that were written by the stage translation direction toward the $-\mathrm{X}$ and $+\mathrm{X}$, respectively. 


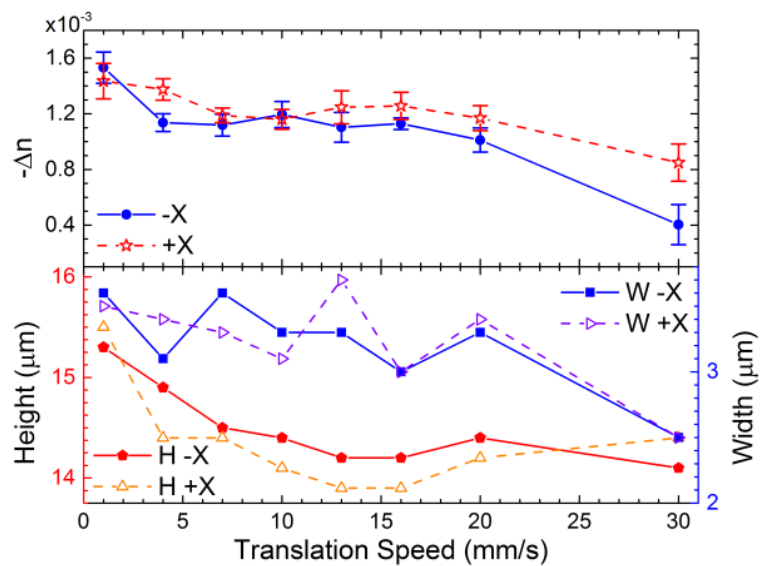

(a)

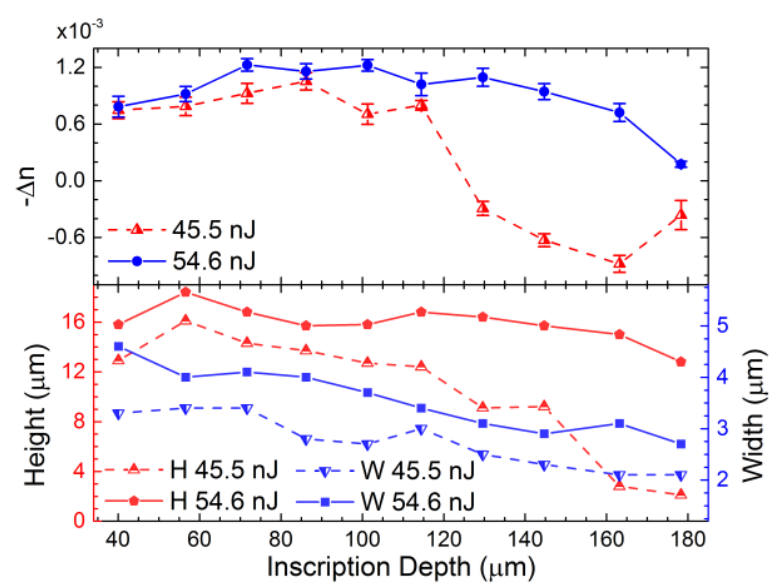

(b)

Figure 4. a) RI contrast (top), and the cross-sectional height and width (bottom) of the tracks as a function of stage's translation speed, where $\mathrm{E}=45.5 \mathrm{~nJ}$ and $\mathrm{D}=85 \mu \mathrm{m}$. b) RI contrast (top), and the cross-sectional height and width (bottom) of the tracks as a function of focus depth, where $\mathrm{S}=10 \mathrm{~mm} / \mathrm{s}$ and $\mathrm{E}=45.5 \mathrm{~nJ}$ or $54.6 \mathrm{~nJ}$. The translation direction was toward $-X$. The RI contrasts were measured at 530-nm wavelength. The error bars represent the variation (standard deviation) in the RI contrast collected from ten different track positions.

and the cross-sectional width and height of the tracks inscribed with varying pulse energies. The translation speed as well as the focus depth were fixed at $10 \mathrm{~mm} / \mathrm{s}$ and $86 \mu \mathrm{m}$, respectively. Linear relations between the pulse energy and RI contrast were fitted to the following equation:

$$
-\Delta n=k \times\left(E-E_{\mathrm{th}}\right) \quad \longrightarrow \quad k(-X,+X)=[2.71,2.44] \times 10^{-2} \mathrm{~nJ}^{-1}, \quad E_{t h}(-X,+X)=[11.09,-0.59] \mathrm{nJ},
$$

where $k$ represents the increasing rate of negative RI contrast $(\Delta n)$, and $E_{t h}$ denotes the pulse energy threshold required to generate the negative RI contrast modification. The differences between the fitting parameters belonging to the $-\mathrm{X}$ and $+\mathrm{X}$ translation directions suggest that our damage tracks subject to the nonreciprocal writing phenomenon, which was previously reported by Yang et al. ${ }^{28}$ using the LRR fs-laser system $(250 \mathrm{kHz}$ at $1064 \mathrm{~nm})$. However, their result showed the directional-dependence behavior only for the tracks written along the Y-axis, unlike our observation that occurred on the X-axis translation. A set of track cross-sections in Figure $3 \mathrm{~b}$ visualizes the so called 'quill pen' structures which was suggested by Kazansky et al. ${ }^{29}$ to be an effect of electron-plasma anisotropic trapping due to a pulse front tilt of the inscribing beam. The observations on our tracks, however, did not show significant differences of track sizes in regard to translation directions. The height-over-width ratios were averagely 4.4 and 4.6 for $-\mathrm{X}$ and $+\mathrm{X}$ directions, respectively. In addition, the ratio tends to increase with the decreasing pulse energy being used in the fabrication process.

Figure $4 \mathrm{a}$ and $4 \mathrm{~b}$ shows the track properties with respect to the variation of the translation speed and the focus depth, respectively. In the range of moderate speed $(5 \mathrm{~mm} / \mathrm{s}$ to $20 \mathrm{~mm} / \mathrm{s})$, it can be seen that the track properties did not change much when the speed was altered. This reflects one of the charteristics of the fs-laser matererial modification in the heat accumlation regim. However when the speed was outside the range, both RI contrast and cross-sectional sizes became either increasing (slower speed) or decreasing (faster speed) by a noticable amount. The focus depth was also one of the paramters that, within a certain degree of variation, the track properties were roughly stable. To be more specific, the tracks inscribed at the depth range between $50 \mu \mathrm{m}$ and $110 \mu \mathrm{m}$ (which was assumed to the minimum-aberration depths of the focusing micro-objective) tended to exhibit only a minor change when the depth was altered. It can also be observed that when the beam was focused beyond the optimal range the tracks generated by the lower pulse energy (red dased line) more suffered to the drops in both RI contrast and sizes. This behavior might be patially due to the material absoptions that attenuated the beam intensity below the strong lasermodification threshold of, for instance, an avalance-electron plasma generation. 


\subsection{Optical Guiding Properties of Inscribed Waveguides before Thermal Treatment}

Some chosen cross-sectional images of pre-annealing waveguides from three different groups are presented in Figure 5a. The insets show the corresponding near-field intensity distributions, captured by the CCD camera and the light source of 1550-nm wavelength. Note that these different-group waveguides were fabricated with the same translation speed/direction and focus depth, but via different schemes of pulse energy alternation (as suggested in the previous section). The observed near-field intensity profiles showed that the fundamental guiding mode was the dominant mode excited in all waveguides presented in this study. Slightly tuning of the relative coupling-stage positions only resulted to the minor changes in mode shape and circularity, but did not lead to the presence of the other higher-order modes. Although the waveguides A05 and B05 were fabricated by using the same initial pulse energy, it can be visually seen that the tracks on the upper and lower rows of B05 were more consistent in sizes and in strength of the modification areas which lead to the higher and more symmetric light confinement (see the insets), compared to A05. In fact, the characterization results revealed that B05's properties $\left(\alpha_{\mathrm{TE}}=1.8 \pm 0.1 \mathrm{~dB} / \mathrm{cm}, \alpha_{\mathrm{TM}}=5.0 \pm 0.2 \mathrm{~dB} / \mathrm{cm}\right.$ and $\mathrm{MDF}=27.6 \pm 1.4 \mu \mathrm{m})$ showed the beam guiding performance better than that of $\mathrm{A} 05\left(\alpha_{\mathrm{TE}}=2.0 \pm 0.2 \mathrm{~dB} / \mathrm{cm}, \alpha_{\mathrm{TM}}\right.$ $=5.6 \pm 0.2 \mathrm{~dB} / \mathrm{cm}$ and $\mathrm{MDF}=28.2 \pm 1.4 \mu \mathrm{m}$ ) which elucidated an explicit benefit of our suggested approach. The same trend also applied to other waveguides fabricated by low pulse energy ( $E_{0}=37.3 \mathrm{~nJ}$ to $\left.48.2 \mathrm{~nJ}\right)$, see Figure $5 \mathrm{~b}$. However, in the high pulse energy range ( $E_{0}=50.9 \mathrm{~nJ}$ to $61.8 \mathrm{~nJ}$ ) Group A waveguides showed less attenuation which might be due to a smaller amount of the high-energy-driven defects and the induced stress field. In comparison to Group A and B, the waveguides in Group C demonstrated significantly higher propagation loss and larger MDF which can be explained by lower RI contrast and smaller sized tracks inscribed on their inner layer tracks. The inferior performance in Group BX might be mainly due to the higher track roughness and the stronger induced stress-field that were typically observed form the damage track fabricated with slower translation speeds.

\subsection{Effects of Heat Treatment}

The same set of tracks (plotted in Figure 3a with -X translation direction) was tested for the effects of heat treatment and the results were plotted in Figure 6a. It was found that the behaviors of RI contrast in response to a sequence of thermal annealing processes were sensitive to the amount of pulse energy being used to generate them. The RI contrasts of tracks generated with low pulse energies ( $<53 \mathrm{~nJ}$, dashed lines) increased after the first three annealing steps (250, 350 and $\left.500{ }^{\circ} \mathrm{C}\right)$, but after the $4^{\text {th }}$ step $\left(700^{\circ} \mathrm{C}\right)$ they started to drop. On the other hand, the RI contrast of the tracks inscribed by high pulse energies (> $53 \mathrm{~nJ}$, solid lines) continued increasing even after the final temperature of $700{ }^{\circ} \mathrm{C}$.

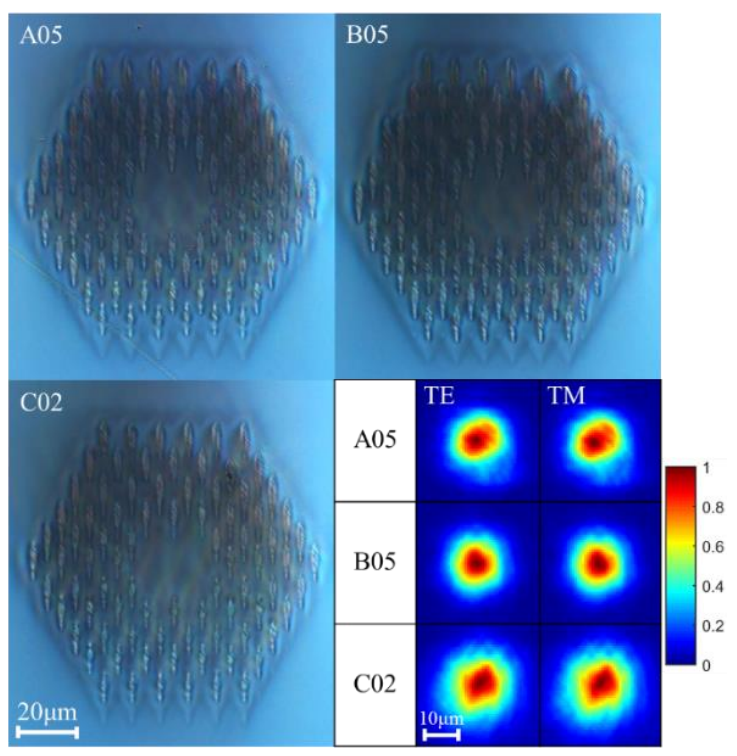

(a)

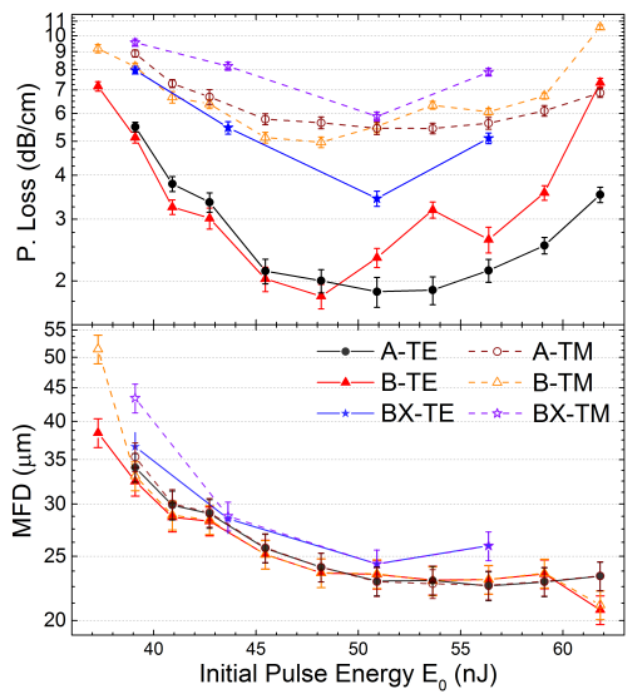

(b)

Figure 5. a) DIC images of optical waveguide cross-sections from different groups of pulse-energy variation schemes. The set of images in the right-bottom corner shows the near-field intensity distribution of waveguide output for TE and TM polarizations at 1550-nm wavelength. b) Propagation loss (top) and mode-field-diameter (bottom) of Group A, B and $\mathrm{BX}$ waveguides before the thermal annealing process as a function of initial pulse energy $\mathrm{E}_{0}$. 


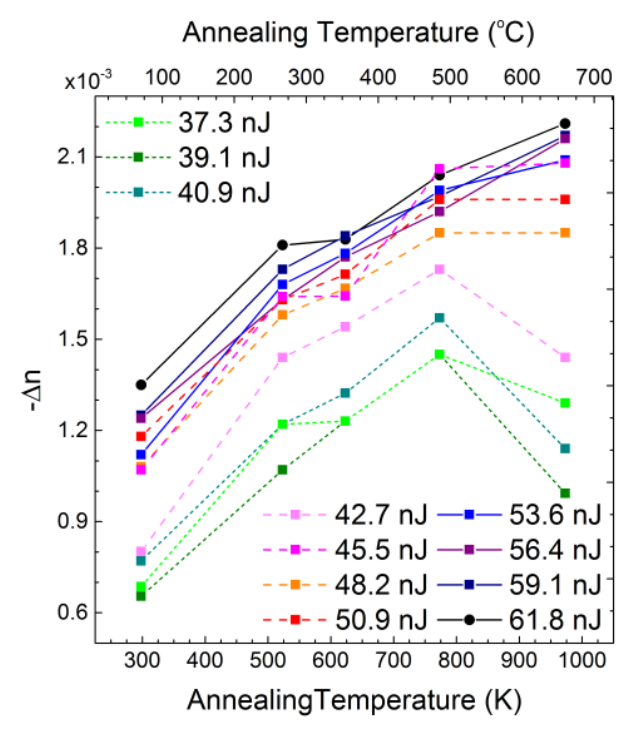

(a)

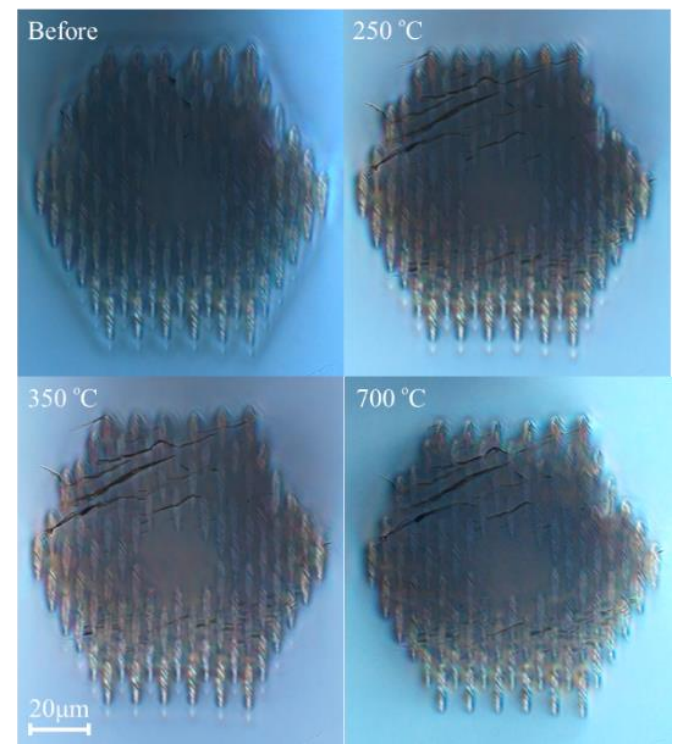

(b)

Figure 6. a) Shows the effects of thermal annealing process to the refractive index contrast measured at 530-nm wavelength of the damage tracks induced by various pulse energies. b) DIC images of waveguide cross-sections captured from B07 waveguide before and after a sequence of thermal annealing processes.

The changes in the cross-sectional sizes via the heat were relatively small (sub micro-meter-scale) and were undeterminable by our optical microscope system with a 100X-magnification objective. It is worth noting that these observations are considerably different from the previous work of Nguyen et al. ${ }^{30}$, where the LRR $(1 \mathrm{kHz}$ at $795 \mathrm{~nm})$ fs-laser was used and the RI contrasts were reported to decrease immediately after the first thermal annealing step at $260{ }^{\circ} \mathrm{C}$. This hence reveals an interesting feature of HRR fs-laser on the fabrication of highly thermal-stable optical structures, which might also give us a clue for the nature of material modifications by HRR fs-laser.

Figure $6 \mathrm{~b}$ shows the cross-sections of waveguide B07 before and after each step of the thermal annealing processes. It can be clearly seen that the stress field induced during the damage track generation as well as non-damage modification area (positive RI contrast) were largely erased after it was thermally treated at $250{ }^{\circ} \mathrm{C}$, and seems to completely disappear after the $700{ }^{\circ} \mathrm{C}$ step. Similar result was demonstrated by Burghoff et al. ${ }^{31}$. However, the lower temperature $\left(200^{\circ} \mathrm{C}\right)$ and the shorter time duration (30 minute) were found to be sufficient in their work to clear the positive RI humps around their damage tracks generated by the LRR fs-laser $(1 \mathrm{kHz}$ at $800 \mathrm{~nm})$. A few shallow surface cracks were observed in our waveguide cladding area, which was assumed to be an effect of the non-uniform expansion/contraction of the radiated and the non-radiated areas during subjected to the heats. These cracks appeared to have no interference to the guided beam which mainly occupied in the waveguide's core.

The optical guiding performances in both TE and TM polarization states at 1550 -nm wavelength were observed to be significantly enhanced by the heat treatments, especially after the first two thermal annealing steps (see Figure 7). The propagation loss tended to decrease to the minimum after the sample was annealed at $350^{\circ} \mathrm{C}$, and then slightly to increase after the higher temperatures of $500{ }^{\circ} \mathrm{C}$ and $700{ }^{\circ} \mathrm{C}$ had been applied. The lowest propagation losses of less than $0.4 \pm 0.1 \mathrm{~dB} / \mathrm{cm}$ and $3.5 \pm 0.2 \mathrm{~dB} / \mathrm{cm}$ for TE and TM, respectively, were achieved from the waveguide B07. This low value of TE propagation loss was calculated from the measured insertion loss of $3.7 \mathrm{~dB}$, the mode-mismatch loss of approximately $1.5 \mathrm{~dB}$ and the double Fresnel loss which was estimated to be $1.4 \mathrm{~dB}$ for the air-and-LN interfaces. The same trend of change regarding the annealing temperatures also applied to MFD, but with a lower turning point i.e. the values of MFD started to raise just after the second annealing step at $350{ }^{\circ} \mathrm{C}$. These improvements of guiding properties might be explained by firstly the increase of light confinement due to the increasing RI contrast (previously shown in Figure 6a), and secondly the reduction of the induced stress-field as well as some transient defects.

Figure 8a shows the wavelength-dependent guiding characteristics of the waveguide B06. It was found that while the propagation loss for TE remained approximately constant over the tested wavelengths, the loss for TM instead changed at around the boundary between the visible and the near-infrared spectrums. The MDF for TE and TM tended 


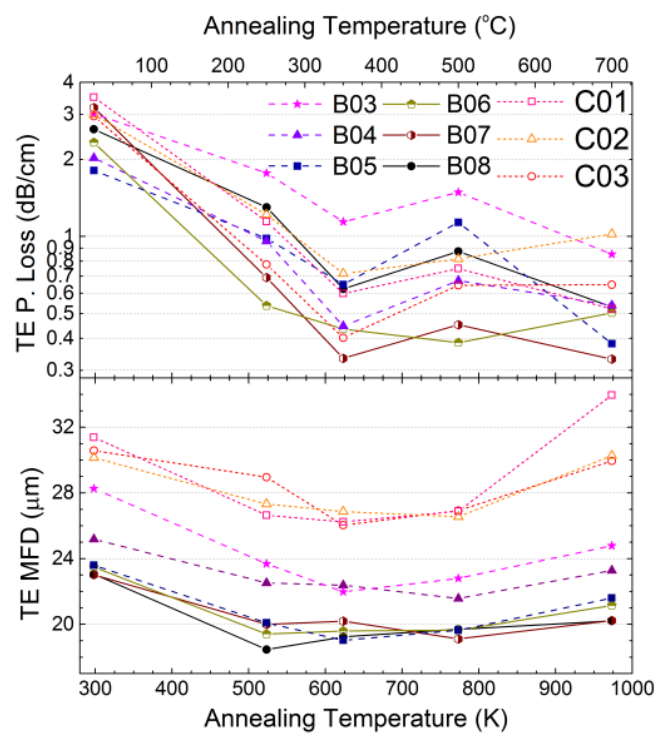

(a)

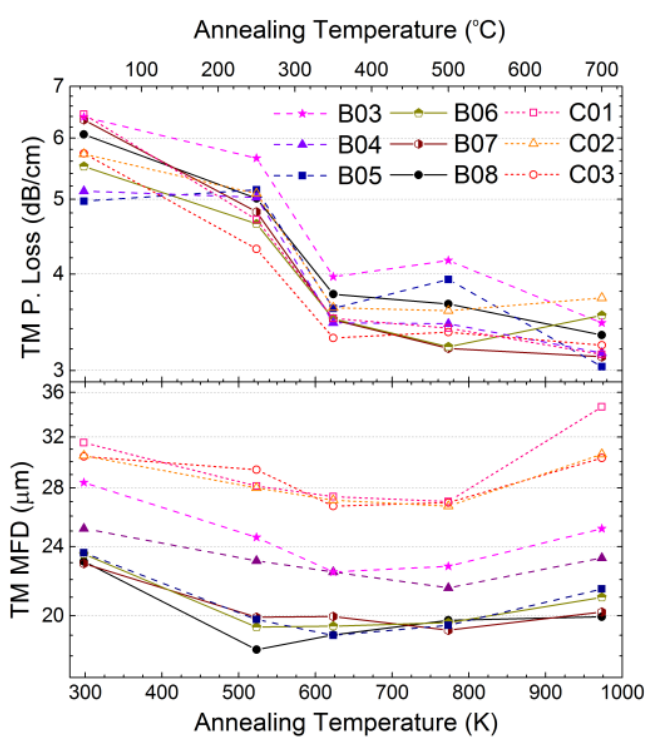

(b)

Figure 7. Propagation loss (top) and mode-field-diameter (bottom) of a set of chosen waveguides before and after a sequence of thermal annealing processes measured at $1550 \mathrm{~nm}$ wavelength and on different polarizations, TE (a) and TM (b).

to increase with the increasing wavelength. Furthermore, numerical simulation of the near-field intensity profile and the MDF as a function of pulse energy were found to be comparable with the experimental result, see Figure 8b. The values of positive RI change for the simulation induced stress-field were varied between $1.0 \times 10^{-4}$ and $3.5 \times 10^{-3}$. Details of the simulation study will be presented elsewhere.

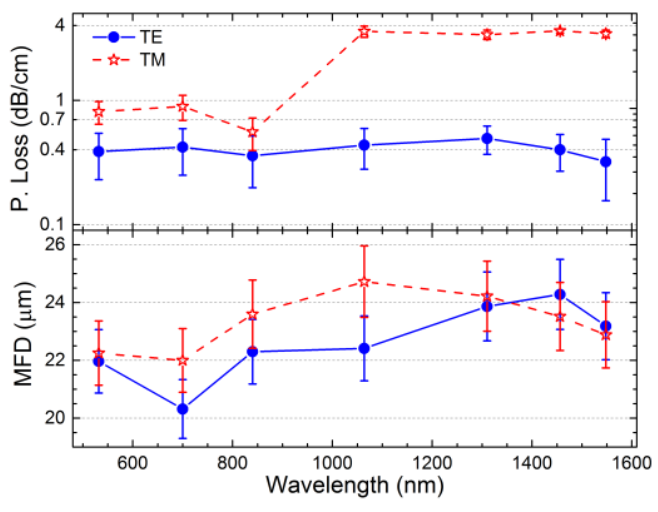

(a)

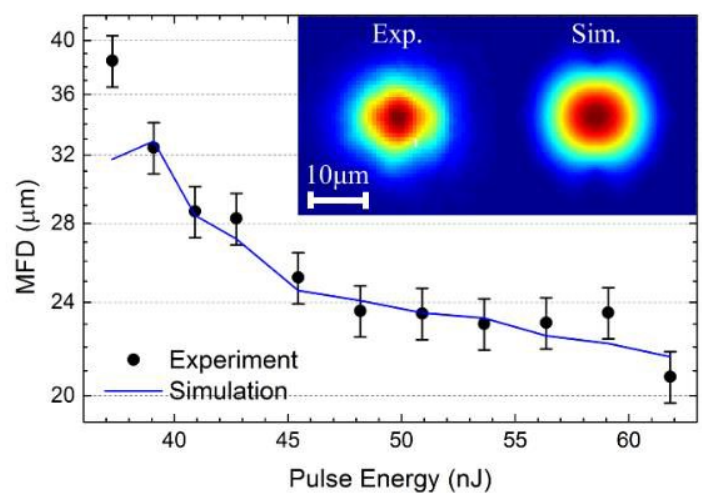

(b)

Figure 8. a) Propagation loss (top) and mode-field-diameter (bottom) of the waveguide B06 after the $2^{\text {nd }}$ thermal annealing step at $350{ }^{\circ} \mathrm{C}$ for 3 hours as a function of input wavelength. b) Comparison of the mode-field-diameters from the experiment and the simulation result of the pre-thermal-annealing Group B waveguides as a function of pulse energy. The considered wavelength was $1550 \mathrm{~nm}$ wavelength and for TE polarization. The insets show the near-field intensity distributions of the waveguide B07 from the experiment (left) and the computer simulation (right). 


\section{CONCLUSIONS}

We have reported on the fabrication and optimization of optical-lattice-like depressed cladding waveguides in zcut 5\%-magnesium-doped LN. The optimized waveguides featured low propagation loss for both the ordinary and extraordinary polarization states and preserved nonlinearity. Single-mode guidance was observed across a wide portion of the spectrum from visible to near infrared $(532 \mathrm{~nm}$ to $1550 \mathrm{~nm}$ ). Two new approaches based on varying the inscribing laser pulse energy in accordance with the position of the tracks were deployed in the fabrication process, which led to enhancement of the waveguide's confinement and intensity distribution symmetry. A large set of RI contrast values and cross-sectional sizes of the damage tracks inscribed with varying operational parameters was presented, which elucidated the effects of the laser pulse energy, the speed and direction of translation stage movement, and the focus depth inside the sample on the fs-laser-induced material modifications in the heat accumulation regime. The heat treatment of the fabricated waveguides revealed an interesting feature of the damage tracks generated by a HRR fs-laser: the RI contrast is enhanced by annealing and remains stable under high temperatures up to $700{ }^{\circ} \mathrm{C}$, opposed to the track degradation that typically occurs in the case of LRR-laser inscription. The low attenuation and high thermal stability of our waveguides suggest promising applications in telecommunications, nonlinear integrated optics, and harsh environment sensors.

\section{ACKNOWLEDGMENTS}

T. Piromjitpong acknowledges the financial support from the Royal Thai Government scholarship.

\section{REFERENCES}

[1] L. Arizmendi, "Photonic applications of lithium niobate crystals," physica status solidi (a) 201(2), 253-283 (2004).

[2] C. Wang et al., "Nanophotonic lithium niobate electro-optic modulators," Optics Express 26(2), 1547-1555 (2018).

[3] A. Rao and S. Fathpour, "Compact Lithium Niobate Electrooptic Modulators," IEEE Journal of Selected Topics in Quantum Electronics 24(4), 1-14 (2018).

[4] R. Luo et al., "On-chip second-harmonic generation and broadband parametric down-conversion in a lithium niobate microresonator," Optics express 25(20), 24531-24539 (2017).

[5] C. Wang et al., "Second harmonic generation in nano-structured thin-film lithium niobate waveguides," Optics express 25(6), 6963-6973 (2017).

[6] M. Bazzan and C. Sada, "Optical waveguides in lithium niobate: Recent developments and applications," Applied Physics Reviews 2(4), 40603 (2015).

[7] S. Gross and M. Withford, "Ultrafast-laser-inscribed 3D integrated photonics: challenges and emerging applications," Nanophotonics 4(3), 332-352 (2015).

[8] J. Burghoff, S. Nolte, and A. Tünnermann, "Origins of waveguiding in femtosecond laser-structured $\mathrm{LiNbO}_{3}$," Applied Physics A 89(1), 127-132 (2007).

[9] A. H. Nejadmalayeri and P. R. Herman, "Rapid thermal annealing in high repetition rate ultrafast laser waveguide writing in lithium niobate," Opt Express 15(17), 10842-10854 (2007).

[10] A. H. Nejadmalayeri and P. R. Herman, "Ultrafast laser waveguide writing: Lithium niobate and the role of circular polarization and picosecond pulse width," Opt Lett 31(20), 2987-2989 (2006). 
[11] R. Thomson et al., "Optical waveguide fabrication in $\mathrm{z}$-cut lithium niobate $\left(\mathrm{LiNbO}_{3}\right)$ using femtosecond pulses in the low repetition rate regime,” Applied Physics Letters 88(11), 111109 (2006).

[12] L. Gui, B. Xu, and T. C. Chong, "Microstructure in lithium niobate by use of focused femtosecond laser pulses,” IEEE Photonics Technology Letters 16(5), 1337-1339 (2004).

[13] J. Lv et al., "Three-dimensional femtosecond laser fabrication of waveguide beam splitters in $\mathrm{LiNbO}_{3}$ crystal," Optical Materials Express 5(6), 1274-1280 (2015).

[14] J. Lv et al., "Femtosecond laser written optical waveguides in z-cut $\mathrm{MgO}$ : $\mathrm{LiNbO}_{3}$ crystal: Fabrication and optical damage investigation,” Optical Materials 57, 169-173 (2016).

[15] V. Guarepi et al., "Bending waveguides made in x-cut lithium niobate crystals for technological applications," Journal of Micromechanics and Microengineering 25(12), 125023 (2015).

[16] Y. Tan, J. R. V. de Aldana, and F. Chen, "Femtosecond laser-written lithium niobate waveguide laser operating at 1085 nm," Optical Engineering 53(10), 107109-107109 (2014).

[17] Y. Liao et al., "Electro-optic integration of embedded electrodes and waveguides in $\mathrm{LiNbO}_{3}$ using a femtosecond laser," Opt Lett 33(19), 2281-2283 (2008).

[18] J. Burghoff et al., "Waveguides in lithium niobate fabricated by focused ultrashort laser pulses," Applied surface science 253(19), 7899-7902 (2007).

[19] S. Kroesen et al., "Monolithic fabrication of quasi phase-matched waveguides by femtosecond laser structuring the $\chi^{(2)}$ nonlinearity," Applied Physics Letters 107(10), 101109 (2015).

[20] H.-D. Nguyen et al., "Heuristic modelling of laser written mid-infrared $\mathrm{LiNbO}_{3}$ stressed-cladding waveguides," Optics express 24(7), 7777-7791 (2016).

[21] M. Bukharin et al., "Techniques of surface optical breakdown prevention for low-depths femtosecond waveguides writing," in Journal of Physics: Conference Series 737(1), 12015 (2016).

[22] J. Qi et al., "Fabrication of polarization-independent single-mode waveguides in lithium niobate crystal with femtosecond laser pulses," Optical Materials Express 6(8), 2554-2559 (2016).

[23] R. He et al., "Femtosecond laser micromachining of lithium niobate depressed cladding waveguides," Optical Materials Express 3(9), 1378-1384 (2013).

[24] S. Kroesen et al., "Electro-optical tunable waveguide embedded multiscan Bragg gratings in lithium niobate by direct femtosecond laser writing," Optics express 22(19), 23339-23348 (2014).

[25] M. Dubov, S. Boscolo, and D. J. Webb, "Microstructured waveguides in z-cut $\mathrm{LiNbO}_{3}$ by high-repetition rate direct femtosecond laser inscription," Optical Materials Express 4(8), 1708-1716 (2014).

[26] S. Gross, M. Dubov, and M. J. Withford, "On the use of the Type I and II scheme for classifying ultrafast laser direct-write photonics," Optics Express 23(6), 7767-7770 (2015).

[27] H. Karakuzu et al., "Optimisation of microstructured waveguides in z-cut $\mathrm{LiNbO}_{3}$ crystals," Optical Materials Express 4(3), 541-552 (2014).

[28] W. Yang, P. G. Kazansky, and Y. P. Svirko, "Non-reciprocal ultrafast laser writing,” Nature Photonics 2(2), 99 (2008). 
[29] P. G. Kazansky et al., “'Quill’ writing with ultrashort light pulses in transparent materials,” Applied physics letters 90(15), 151120 (2007).

[30] H.-D. Nguyen et al., "Low-loss 3D-laser-written mid-infrared $\mathrm{LiNbO}_{3}$ depressed-index cladding waveguides for both TE and TM polarizations," Optics Express 25(4), 3722-3736 (2017).

[31] J. Burghoff et al., "Structural properties of femtosecond laser-induced modifications in $\mathrm{LiNbO}_{3}$," Applied Physics A 86(2), 165-170 (2007). 